\title{
LA GESTIÓN DEL CONFLICTO EN EL ÁMBITO LABORAL DE LAS ORGANIZACIONES TURÍSTICAS: UNA HERRAMIENTA DE MARKETING INTERNO
}

\author{
M.C. PARADINAS \\ Esic, Business \& Marketing School/Doctorate URJC \\ carmen.paradinas@esic.edu
}

Submetido em 31/01/2019 e aceito em 14/02/2020

DOI: $10.15628 /$ holos. 2020.8260

\begin{abstract}
RESUMEN
El objeto de este estudio es realizar una revisión bibliográfica que permita conocer el estado del arte en cuanto a la gestión del conflicto en el ámbito laboral de las organizaciones turísticas y su relación con las herramientas empleadas por éstas para fomentar el sentimiento de pertenencia de sus empleados a la organización y a su vez que los mismos puedan ser embajadores de marca. Así, los programas de prevención y gestión de los conflictos surgidos entre las personas empleadas por la organización pueden enmarcarse dentro de las herramientas llevadas a cabo por las empresas en el entorno del marketing interno o "employer breanding" interno y, de esta forma, crear una importante ventaja competitiva frente a los
\end{abstract}

competidores. Para ello se ha procedido al análisis de tesis doctorales, publicaciones de investigación y divulgación científica, libros y sitios previamente contrastados para amparar su fiabilidad. Introduciendo diversos criterios de búsqueda selectiva que hicieron posible extraer los documentos más relevantes para la investigación. Los resultados sugieren que, aunque hay numerosos estudios relacionados con la gestión del conflicto en las organizaciones, son pocos, comparativamente hablando, los que se focalizan en el sector turístico. Igualmente se observa que es escasa la bibliografía que analiza la prevención y gestión del conflicto como una herramienta relevante en cuanto al marketing interno.

PALABRAS CLAVE: Gestión del conflicto, marketing interno, ámbito laboral, organizaciones turísticas, ventaja competitiva.

\section{CONFLICT MANAGEMENT IN THE WORK PLACE OF TOURISM ORGANIZATIONS: AN INTERNAL MARKETING TOOL}

\begin{abstract}
The aim of this study is to carry out a bibliographic review that will allow us to know the state of the art regarding the conflict management in the working environment of tourist organizations and its relation with the tools used by them to promote the feeling of belonging of their employees to the organization and at the same time that they can be brand ambassadors. In this way, the prevention and management programmes for conflicts arising between people employed by the organisation can be framed within the tools carried out by companies in the internal marketing environment or internal employer breanding and, in this way, create an important competitive advantage over competitors. To this end, the
\end{abstract}

analysis of doctoral theses, research publications and scientific dissemination, books and previously contrasted sites has been carried out in order to protect their reliability. Introducing various selective search criteria that made it possible to extract the most relevant documents for research. The results suggest that, although there are numerous studies related to the management of conflict in organisations, comparatively few focus on the tourism sector. It is also observed that there is a scarce bibliography that analyzes conflict prevention and management as a relevant tool in terms of internal marketing.

KEYWORDS: Conflict management, internal marketing, working environment, tourism organizations, competitive edge. 


\section{INTRODUCCIÓN}

Este trabajo trata de estudiar la visión que tienen las organizaciones y, en concreto, las relacionadas con el sector turístico, de los conflictos que se dan en su seno, cómo lo gestionan y cómo lo afrontan cuando el mismo ha surgido entre sus empleados.

Para ello se ha realizado una revisión del estado actual de tema, analizando el impacto que el conflicto entre el personal empleado en los hoteles puede tener de cara a los clientes, qué sistemas de prevención del conflicto emplea la organización, qué canales utiliza para su gestión y cómo estas actuaciones se enmarcan dentro, no sólo de las políticas de responsabilidades social corporativa, sino también dentro de las herramientas de marketing interno o employer branding interno que la entidad desarrolla de cara a la satisfacción de sus empleados.

Las organizaciones están compuestas por personas y por lo tanto los conflictos y las crisis son endémicos, resultando lo importante atender a cómo se gestionan en los niveles de personal y organizacional, teniendo en cuanta que algunos de esos conflictos pueden ser agudos y otros crónicos (Fried, 2011).

Es relevante para afrontar el estudio desde una perspectiva holística, destacar el hecho de que no todo conflicto es susceptible de solucionarse y por ello el análisis del mismo no debe centrarse sólo en las vías de resolución del mismo sino que, ante la referida circunstancia, debe afrontarse también el estudio de su gestión por cuanto, en ocasiones, afloran conflictos en el seno de la organización, ya sean intergrupales o intragrupales (Benítez, Medina y Munduate, 2012) que, si bien no se resuelven, pueden ser gestionados de forma que no influyan en la productividad del empleado, del equipo y en general de las partes implicadas y así no impacte directamente en los costes de la empresa.

Teniendo esto en cuenta, el término gestión de conflictos es más amplio que el de resolución de conflictos tal y como señalan Yirik, Yildirim y Çentinkaya (2015), ya que la resolución abarca la reconciliación de conflictos, mientras que la gestión de los mismos, engloba no solo la reconciliación sino cómo dirigir los conflictos destructivos hacia conflictos con resultados constructivos. Es por esto que el concepto de "resolución" de conflictos, que siempre se ha entendido como eliminación del enfrentamiento, crece para añadir también a su ámbito, no solo la "gestión" del mismo sino también la identificación de los intereses de las partes implicadas y aislándolas de sus posiciones (Fajardo, 2015).

Las organizaciones turísticas se han visto influenciadas en los últimos años por nuevas necesidades en cuanto a gestión de personas se refiere, constatándose la existencia de nuevas fórmulas y procedimientos que provocan en las que las utilizan, un posicionamiento preferente al frente de esos cambios generando ventaja competitiva.

La principal novedad es posicionar a la persona, es decir a quien trabaja para la organización, en el centro de la misma siendo una de las herramientas más utilizadas aquella que, siguiendo el criterio de la persona como centro, se preocupa de alinearla con la cultura empresarial y así conseguir empleados comprometidos y embajadores de marca. Dicha herramienta es el employer branding interno o marketing interno, empleada por las organizaciones que no sólo enfocan sus esfuerzos en el marketing externo sino que entienden que su personal es tan importante o más que sus clientes, proveedores y demás steakholders. 
Por ello, si las personas que componen la organización se encuentran en un ambiente conflictivo, no se sienten escuchados ante una situación de conflicto y el entorno es desfavorable a la conversión del mismo en creatividad y oportunidad, entre otras, cosas porque no forma parte de la cultura empresarial o la misma no se ha transmitido, se desencadenarán una serie de hechos perjudiciales para la empresa no solo de cara al cliente sino que se verán reflejados en los costes.

\section{REVISIÓN BIBLIOGRÁFICA}

\subsection{Aproximación al concepto de conflicto.}

Se pueden encontrar multitud de definiciones para a palabra conflicto, siendo importante resaltar que, dependiendo de la acepción que se tome de éste, su afrontamiento se realizará de una forma u otra, y el modo de afrontar estas situaciones influye, no solo en los posibles beneficios de la empresa o las condiciones laborales, sino también en la calidad y salud de la propia estructura empresarial. (Merino, 2008).

Tradicionalmente el conflicto ha tenido una acepción negativa entendido como guerra, enfrentamiento, controversia, lucha, combate (París, 2003). De esta manera, culturalmente, creemos que si nos encontramos en una situación de conflicto con alguna persona o grupo de ellas, ésta dará lugar a un combate o pelea donde sólo puede haber vencedores y vencidos (Die, 2013).

Hocker y Wilmot (2014) definen el término como "una lucha que se expresa al menos entre dos partes independientes que perciben que los otros poseen metas, recursos escasos $e$ interferencias incompatibles que les impiden a ellos alcanzar las suyas", si bien para Coser (1956), "el conflicto es una lucha sobre valores o reclamo de estatus, poder y recursos escasos en los cuales el objetivo de una de las partes en conflicto es no solamente obtener valores deseados sino también perjudicar o eliminar a sus rivales".

Se han puesto de manifiesto las definiciones más utilizadas por voces expertas en el estudio del conflicto, pudiendo observarse que se constata en todas ellas que las partes implicadas en el conflicto perciben que sus intereses están siendo afectados o pudieran estarlo, que sus metas son incompatibles y que si el logro de las mismas se ve afectado, desencadenará en conflicto (González 2010).

Derivada de esta acepción negativa del conflicto, podemos encontrar numerosas definiciones todas ellas en el mismo sentido (pelea, angustia, batalla,...), pero si realmente el interés de las partes es resolver el conflicto, resultará más adecuado emplear el término conflicto desde una perspectiva positiva y entenderlo como una situación enriquecedora, y es desde este punto de vista, desde el que los estudiosos del conflicto prefieren verlo. Conflicto como oportunidad de aprender, de crecer, de desarrollar nuevos paradigmas que impulsen a las partes hacia el crecimiento personal y social (Gómez, 2013).

\subsection{El conflicto organizacional.}

La aparición de situaciones de conflicto en el seno de la organización conlleva unas consecuencias a distintos niveles que no solo afectan a las personas que la componen, sino también a la propia organización creando una serie de costes tanto emocionales como económicos. 
Las discrepancias surgen de las relaciones que se entablan entre los equipos profesionales, bien sea entre iguales, bien sea entre personas con distintas responsabilidades dentro de la empresa, y por ello será necesario entender el conflicto considerando los factores que alteran su normal funcionamiento, debiendo analizar sus causas o motivos antes de intentar abordarlo. Teniendo en cuenta la clasificación establecida por Moore (1986), encontraríamos diversos tipos de conflicto dentro de una organización, dependiendo de sus causas:

Tabla 1: Tipos de conflicto dentro de la organización según sus causas

\begin{tabular}{|c|}
\hline Conflictos \\
\hline Sobre intereses \\
De información \\
De relaciones \\
De valores \\
De estructura \\
\hline
\end{tabular}

El modelo de clasificación expuesto en la tabla 1 ha sufrido adaptaciones como la de Laca y Alzate (2004) que suprimen el conflicto de información por entender que en cualquier conflicto el intercambio de información entre las partes desaparece o se distorsiona por lo que el conflicto de información resultaría inherente a cualquier tipo de conflicto. Y otros autores como Redorta (2004) amplían el espectro identificando un mayor número de conflictos en base a que los mismos siguen patrones de comportamiento reconocibles e identificables:

Tabla 2: Tipos de conflicto en base a patrones de comportamiento

\begin{tabular}{|c|}
\hline Conflictos de \\
\hline Recursos escasos \\
Poder \\
Autoestima \\
Valores \\
Identidad \\
Expectativas \\
\hline Inadaptación \\
Información \\
Intereses \\
Relaciones personales \\
Inhibición \\
Legitimación \\
Normativos \\
Atributivos \\
Estructurales \\
\hline
\end{tabular}


Además de identificar el tipo de conflicto ante el que se encuentran las partes para poder gestionarlo de forma efectiva y sobre todo positiva, es necesario reconocer de qué forma lo afrontarán los intervinientes. Para ello se puede atender a la clasificación de Rahim (1985) en la que partiendo de dos variables que aparecerán con mayor o menor intensidad: preocupación por los demás y preocupación por sí mismo, establece cinco formas de afrontar el conflicto:

- Acomodativo (se centra en tratar de satisfacer las preocupaciones de otro prestando poca atención o ninguna a las propias)

- Colaborativo (trabaja con la otra parte de forma cooperativa para tratar así de encontrar una solución que integre y satisfaga las preocupaciones de todos)

- Evitativo (muestra falta de interés y atención hacia las preocupaciones de cualquiera de las partes, mostrando apatía, negligencia o incluso retirándose)

- Competitivo (trata de ganar en sus propios interese a expensas de los intereses y preocupaciones de los demás)

- Compromiso (refleja una actitud pragmática prefiriendo encontrar una solución mutuamente aceptable que satisface parcialmente los intereses de ambas partes).

Dado que el conflicto en las organizaciones se está convirtiendo en un aspecto a tener en cuenta y de interés, el análisis del mismo tomando en consideración las variables referenciadas ayuda a realizar un mapa del conflicto que permita gestionarlo de una manera eficaz y efectiva.

Si bien hasta hace relativamente poco tiempo los conflictos en el ámbito laboral eran considerados meros incidentes desagradables (Carnegie, 2011), hoy las empresas son conscientes de que las consecuencias son importantes y provocan costes económicos y emocionales por lo que se hace necesario implantar técnicas de prevención y gestión del conflicto con el fin de evitarlos y además generar así importantes ventajas competitivas ya que la falta de atención al conflicto puede generar graves situaciones si se permite o, no se controla la escalada del mismo, tales como constantes discusiones, bajas laborales o incluso agresiones o mobbing, ya que los conflictos surgidos entre el personal de una organización por causas personales, interpersonales o derivados de la estructura y cultura de la propia organización son fuente de grandes pérdidas económicas y humanas (Baixauli, 2017).

Por tanto, si un conflicto en el entorno laboral puede derivar en una baja, es claro que la misma tiene un coste no solo para la empresa sino también para la Administración Pública y además si aquel no se gestiona correctamente, puede desembocar en salidas de los empleados de la empresa, provocando una alto rotación con los costes que ello conlleva (nuevos procesos de selección, formación, etc).

2.3 Marketing interno o employer branding interno. La mediación como herramienta de gestión del conflicto.

¿Cómo pueden las organizaciones evitar las referidas bajas del puesto de trabajo o la disminución de la productividad por el tiempo que las partes afectadas por el conflicto tienen que dedicar a su gestión?

Es evidente que implantando sistemas de prevención y gestión del conflicto, como ya se ha expuesto con anterioridad, que favorecerán una plantilla más eficaz y eficiente y sobre todo que 
se encuentra cómoda, atendida, entendida e implicada en la búsqueda de soluciones ante los conflictos de una forma cooperativa.

¿Qué se consigue además con ello? Que el personal se sienta alineado con la cultura empresarial y de esa forma, comprometido con la misión, visión y valores de la empresa. Ello provocará, no sólo que se sientan orgullosos de trabajar en ella sino que la recomienden a otras personas.

En este punto resulta interesante analizar el término de reciente nuevo cuño: Internal Branding. Según afirman De la Guardia y Enrique (2017), se trata de un procedimiento que se centra especialmente en establecer una relación de lealtad entre el empleado y la marca de su empresa, lo cual tiene como objetivo aumentar su compromiso y por lo tanto retener al mejor talento. Si la empresa es capaz de transmitir fortaleza en su marca al personal, éste estará motivado y por lo tanto persuasivo hacia el cliente y ello será posible si atiende a las habilidades, conocimientos y aptitudes de sus empelados, es decir no solo a sus habilidades cognitivas, sino a las no cognitivas que son las que más influyen a la hora de enfrentarse al conflicto. Centrándose en ello, se podrán potenciar las áreas de mejora intentando alinearlas con la estrategia de la empresa, lo que al final será transmitido al cliente externo.

En las últimas décadas, los factores intangibles de las organizaciones han ido cobrando especial importancia a la hora de posicionarse en el mercado (Grant, 1996). Por ello, la valoración de la experiencia que el cliente perciba de estos intangibles resulta de gran interés.

Tradicionalmente se ha entendido la forma de resolver los conflictos como un proceso en el que resulta una parte ganadora y otra vencedora, lo que limita el abanico de soluciones posibles y dificulta o destruye la relación entre las partes involucradas generando además, no solo unos costes económicos y sociales, sino, y lo que es más importante para la organización en cuanto a su personal se refiere, unos costes emocionales, relacionales y afectivos importantes.

Se puede encontrar un paradigma contrario a la fórmula ganar/perder que es el de ganar/ganar, que es empleado cada vez con más frecuencia en las organizaciones especialmente para conflictos que podríamos denominar informales. Esta fórmula es aplicada en lo que se conoce como Métodos de Resolución Alternativa de Conflictos (ADR, Alternative Dispute Resolutions) que consisten en mecanismos no formales que aportan un elemento fundamental como es la humanización del conflicto interviniendo una tercera persona que actúa como canalizador de la comunicación o facilitadora, especialista en resolución y prevención del conflicto (Fuquen, 2003).

Es importante diferenciar cada una de las técnicas de ADR ya que en muchas ocasiones el abordaje del conflicto y su resolución, pueden llegar a fracasar, no tanto por el tipo de conflicto o las partes que intervengan en él, sino por no elegir la vía adecuada.

\section{MEDIACIÓN}

A través de la mediación, las partes en conflicto acuden de forma voluntaria a un proceso en el que una tercera persona, independiente e imparcial, les ayuda a comunicarse para que puedan llegar a comprender realmente sus diferencias, determinar el origen y las consecuencias de la falta de acuerdos. De esta manera, esa tercera persona mediadora, les ayuda a confrontar su visión de la disputa y a encontrar soluciones por si mismos para evitar acudir a la vía judicial, siempre en un espacio de diálogo, respeto, igualdad y libertad. 
Se trata de un procedimiento confidencial, voluntario, ágil y mucho menos costoso que un procedimiento judicial, tanto económica como emocionalmente al permitir el mantenimiento o mejora de las relaciones futuras entre las partes y llegar a acuerdos que, de acudir a la vía judicial, no se tratarían ni alcanzarían.

\section{NEGOCIACIÓN}

A lo largo del presente manual encontraremos varias definiciones de negociación sin que ello suponga que unas excluyan a otras.

En relación al conflicto e independientemente del ámbito en el que se produzca, podemos entender por negociación aquel proceso a través del cual dos partes con posiciones en conflicto, se unen de forma voluntaria para establecer unas pautas comunes de intercambio con el fin de intentar satisfacer los intereses de ambas de una forma satisfactoria. Esa unión se puede dar entre las propias partes o entre quienes ellas mismas decidan que puedan actuar en su representación.

\section{ARBITRAJE}

El arbitraje es un proceso empleado para resolver un litigio evitando con ello acudir a la vía judicial, en el que las partes de forma voluntaria y de común acuerdo, deciden someter la resolución de sus diferencias a la decisión de un tercero independiente, el árbitro, o a un tribunal arbitral, que será el encargado de dar solución al conflicto a través del dictado de un laudo arbitral.

\section{Existen dos tipos de arbitraje:}

Arbitraje de Derecho, en el que el árbitro que va a resolver el conflicto es un experto en derecho, pero ajeno a la jurisdicción ordinaria, y lo hará en base a la aplicación de las leyes.

Arbitraje de equidad, en el que el árbitro que va a resolver el conflicto no aplica leyes ni reglamentos sino su leal saber y entender de lo que es justo y equitativo, tratándose habitualmente de un experto en la materia objeto de controversia.

Tras esta breve definición de cada uno de los métodos de ADR, es conveniente resaltar que, dadas las características del conflicto que se da en el entorno laboral, resulta recomendable el empleo de la mediación como herramienta de prevención y gestión del conflicto, la cual puede ser realizada o por miembros de la propia organización previamente formados para ello o por un servicio externo especializado en procesos de mediación. Y es la más adecuada porque en dichos procesos, las partes abordan el conflicto desde su origen, no buscando necesariamente su resolución sino a veces simplemente tratando de aprender a convivir con el conflicto, lo cual en el entorno laboral resulta muy conveniente.

De esta manera la organización podrá emplear como herramienta de marketing interno la prevención y gestión del conflicto a través de la mediación por cuanto las partes implicadas alcanzan sus acuerdos de forma voluntaria y por tanto el acuerdo al que se llegue va a ser a través del consenso (San Cristobal, 2013), lo que va a permitir y fomentar un ambiente de diálogo, cooperación e interés por la persona. 
2.4. El conflicto en las organizaciones turísticas y la importancia de su gestión ante la influencia del turismo en el PIB de Portugal.

En Portugal, el turismo representa una actividad económica de gran relevancia, con un enorme potencial de crecimiento, en términos de riqueza y empleo (Mendes y Vareiro, 2012). Según Lopes (2010, citado en Mendes y Vareiro, 2012), el turismo revela su importancia no sólo en relación con el Producto Interior Bruto (PIB), sino también en relación con el empleo total.

El consumo del turismo en el territorio portugués llegó al 13,7\% del PIB en el año 2017 (un 14,5\% más que en 2016), lo que se traduce en 26.700 millones de euros, según datos de la "Conta Satélite do Turismo" divulgados por el Instituto Nacional de Estatística portugués.

Dada la importancia del sector turístico en el crecimiento y desarrollo del país, resulta especialmente relevante el estudio de las relaciones que se establecen entre las personas que integran una organización turística, por cuanto que el componente de la diversidad es un factor clave para el desarrollo de programas y actuaciones encaminadas a la resolución y/o gestión de conflictos. Estos conflictos afectan a la estructura de la propia organización y a la imagen que ésta proyecta de cara al cliente cuando las relaciones personales y profesionales se encuentran en un estado de desgaste sin que se afronte la situación o se afronte de forma incorrecta.

Conde y Amaya (2007) hacen referencia a la parte intangible reconocida dentro de lo que denominan producto hotelero considerando que el mismo "está formado por el conjunto de bienes y servicios que se ofrecen en el mercado, para el confort material y espiritual, en forma individual o en una gama muy amplia de combinaciones resultantes de las necesidades y deseos del consumidor al que le llamamos turista.".

De esta forma, un producto como el hotelero se puede definir como un conjunto específico de atributos que aporta al comprador, no solo el servicio de base característico de la clase de producto, sino también un conjunto de servicios secundarios, que constituyen elementos distintivos, susceptibles de influir en las preferencias de los compradores (Lambin, 1995 en Conde y Amaya, 2007).

Así, se puede inferir que existe una estrecha e importante relación entre el clima de servicio y la percepción del cliente, ya que si ese clima no es bueno, será transmitido de forma ineludible a la relación entre empleado y usuario del servicio/consumidor del producto hotelero.

En los últimos años, la calidad del servicio se ha convertido en uno de los temas centrales de preocupación para la dirección de las empresas, llegando incluso a incorporar sus excelencias en las correspondientes campañas de Marketing, especialmente por su capacidad para generar ventaja competitiva (Carmeli, 2008; García, 2001).

Es importante que las organizaciones turísticas tomen conciencia de que la innovación no sólo es exclusiva de los productos tangibles o servicios y puede extenderse al propio proceso, a la organización o a la gestión del marketing (Barbosa y Dominique - Ferreira, 2012) y que para implantar nuevos procesos de gestión del conflicto a veces, es necesario cambiar la cultura empresarial. En la materia que nos ocupa, estaríamos hablando inevitablemente de innovación, de procesos, no de producto ya que el término innovación de procesos engloba la previsión de nuevas estrategias laborales, la actividad de proceso y la implantación del cambio en las complejas dimensiones humanas, tecnológicas y organizacionales de la empresa, buscando esta última, el desempeño. (Devenport, 1993 en Barbosa y Dominique - Ferreira, 2012). La innovación de 
procesos es típicamente aplicadas en la organización mientras que la de producto o servicio está orientada al mercado (Freire, 2000).

\section{METOdOLOGÍA}

Este trabajo se ha comenzado a desarrollar mediante una revisión bibliográfica de la literatura al tratarse del inicio de una investigación relativa a la gestión del conflicto en las organizaciones, en concreto en el ámbito laboral del sector turístico hotelero.

La metodología empleada para esta investigación ha sido una revisión bibliográfica de carácter sistemático de la materia objeto de estudio.

En primer lugar se ha procedido a la definición del problema para así, poder realizar una búsqueda bibliográfica acertada y que resulte de aportación. Para ello se ha trabajado sobre el concepto conflicto y la resolución, prevención y gestión del mismo en un primer momento, para después reducirlo al ámbito de las organizaciones y posteriormente centrarse en las de carácter turístico.

En esta revisión se han analizado tesis doctorales, publicaciones de investigación y divulgación científica, libros y sitios previamente contrastados para amparar su fiabilidad. Introduciendo diversos criterios de búsqueda selectiva que hicieron posible extraer los documentos más relevantes para la investigación.

Se han empleado criterios de búsqueda en inglés y en español tales como: Organizational Conflict, Conflict Resolution, Conflict Mediation, Employee Conflict, Effective Conflict, Conflict Management, Tourism Conflict Management, Labor Conflict in Tourism Management o Conflicto Organizacional, Gestión de Conflictos, Resolución de Conflictos Laborales, Trabajo Emocional e el Sector Turístico y Conflicto Laboral en el Sector Turístico.

Posteriormente, se han introducido los referidos criterios de búsqueda en las siguientes bases de datos: Teseo, Academic Search Complete, JCR - Journal Citation Report, SCOPUS, Science Direct; Web of Science, ABI, Google Académico y Redalyc todas ellas relacionadas con las Ciencias Jurídicas y Sociales y Humanidades.

La introducción de dichos criterios arrojó un resultado de más de quinientos artículos eligiéndose para su estudio únicamente los publicados a partir del año 2000, si bien en este artículo se citan autores cuyas publicaciones son anteriores ya que los mismos son reiteradamente citados por los autores cuya bibliografía se ha revisado y se ha considerado necesario incluirlos al tener gran peso en el ámbito de la gestión del conflicto y especialmente en el ámbito organizacional.

Por último, se ha procedido al análisis de la información atendiendo a los documentos considerados más relevantes y útiles, si bien esta fase se ha realizado en paralelo a la primera por ser un proceso constante durante el desarrollo de la investigación.

\section{RESULTADOS Y CONCLUSIONES}

Al analizar el conflicto, desde la teoría crítica, se aprecia cómo es catalogado como algo natural, inherente a las relaciones humanas y naturalmente por tanto a todo tipo de organizaciones. 
La gestión de los conflictos no implica necesariamente evitarlos, reducirlos o terminar con ellos sino que tiene que ver con diseñar estrategias efectivas que minimicen las alteraciones que provocan y mejoren las funciones constructivas para que se alcance el aprendizaje colectivo y mejoren la eficacia de una organización (Rahim, 2002).

Partiendo de esas premisas, el objetivo de este trabajo ha sido investigar la influencia del conflicto en el marco de las organizaciones y en concreto de las turísticas y cómo este impacta sobre la calidad de los servicios, el clima en la organización y el sentimiento de pertenencia y compromiso del personal contratado. Estos factores son relevantes a la hora de rebajar costes en la empresa si se previene y gestiona el conflicto con las herramientas adecuadas tras un correcto análisis y diagnóstico de la situación; costes derivados de las bajas laborales que puede provocar, el absentismo, el tiempo que las partes implicadas deben dedicar a resolverlo en detrimento de su productividad, amplia rotación, lentos y costosos procesos de selección y formación, etc.

Todo ello provoca, no solo unos elevados costes económicos sino emocionales, que dan pie a un empeoramiento del clima del servicio que redunda en el cliente y es fácilmente percibido por él.

Las organizaciones que invierten en innovación, no solo de producto sino también de procesos adquieren una mayor ventaja competitiva y dentro de esos procesos se puede incluir la gestión del conflicto. Esta innovación requiere formar al personal para trabajar no solo en sus habilidades cognitivas, sino también en las habilidades no cognitivas necesarias en los procesos de gestión del conflicto atendiendo a sus áreas de mejora y dotándoles de herramientas de prevención, gestión y resolución que permitan detectar el conflicto a tiempo y tratarlo de manera que no escale hasta el punto de hacerse inmanejable y que vaya en detrimento de la organización.

Una de esas herramientas es la utilización de la mediación para la resolución del conflicto al tratarse de un proceso en el que las partes implicadas, llegan por si mismas a acuerdos que resuelven su conflicto o, en los casos en que ello no sea posible, por lo menos aprenden a gestionarlo y a convivir. Además de llegar por si mismas a esos acuerdos, son acompañadas por una tercera persona que le asiste en el proceso facilitando al comunicación, persona que a través de una correcta formación, puede ser un miembro de la organización o y profesional externo.

Si se actúa así, las personas que trabajan en la empresa estarán más motivadas, serán más productivas y al verse capaces de resolver los conflictos por si mismos con los medios y herramientas facilitados por la organización, se sentirán comprometidos con los valores de ésta. Ello será percibido por el cliente quien recibirá un servicio de alta calidad.

Cada vez es mayor la tendencia de las empresas a poner a la persona en el centro apostando por técnicas y herramientas que hagan que las personas que trabajan en ella se sientan parte de su filosofía y estén alineados con la estrategia a seguir. Por ello, ya no se centran todos los esfuerzos en el marketing externo sino también en el interno, con el fin de evitar la rotación y retener el talento en la organización. Si el personal siente que trabaja en una empresa saludable y comprometida con sus necesidades y emociones, también mostrará ese compromiso.

El conflicto en la mayoría de las ocasiones provoca en las personas una cierta alteración, inquietud e inseguridad pero si el empleado se encuentra arropado por su organización ante estas situaciones porque le ha proporcionado las herramientas adecuadas para la gestión del conflicto al que se enfrenta, será un buen embajador de marca y esa oportunidad debe ser aprovechada por 
el departamento de recursos humanos a la hora de promocionar su empresa desde el punto de vista interno junto con el departamento de marketing.

\section{REFERENCIAS BIBLIOGRÁFICAS}

ALVARADO, F. y ELINA. M. (2003). "Los conflictos y las formas alternativas de resolución". Tabula Rasa [en linea] 2003, (enero-diciembre): [Fecha de consulta: 31 de enero de 2019] Disponible en:<http://www.redalyc.org/articulo.oa?id=39600114> ISSN 1794-2489

BAIXAULI, E. (2017). “La mediación empresarial. Una herramienta para la prevención del mobbing”. Madrid, SEPIN.

BARBOSA, B.M y DOMINIQUE - FERREIRA, S. (2012). "La innovación de los procesos. Diferenciación en los Servicios turísticos". Estudios y Perspectivas en Turismo. Vol. 21, págs.: 963 - 976.

BENÍTEZ, M., MEDINA, F. J., MUNDUATE, L. (2012) "La gestión de conflictos relacionales en las organizaciones de servicios". Anales de Psicología 2012, Vol. 28, n 1, págs.: 139 - 149. Servicio de Publicaciones de la Universidad de Murcia.

CARMELI, A. (2008). "Top team behavioural integration and the performance of service organizations". Group \& Organization Management, 33, 712-735.

CARNEGIE, D (2011): "Las cinco habilidades esenciales para tratar con las personas". Ediciones Elipse.

CONDE, E.M. y AMAYA, C.M (2007). "El producto hotelero: visto como un conjunto de atributos tangibles e intangibles". Gestión Turística, no 8, págs.: 75 a 84.

DE LA GUARDIA, C y ENRIQUE, A. (2017): "Revisión del concepto de internal branding: la transmisión de la promessa de marca a los clientes por parte de los empleados". Revista Gráfica. Vol. 5, no 9, págs. 29 - 37.

DIE, F. (2013). "Análisis de las necesidades jurídicas y metodológicas para la implementación de sistemas ADR en centros hospitalarios públicos" [Tesis Doctoral]. Universidad Complutense de Madrid.

FAJARDO, P. (2015): “Cooperar como estrategia. Sobre el uso de métodos alternativos para la solución de los conflictos de las empresas". [Tesis Doctoral]. Universidad Pontificia de Comillas.

FREIRE, A. (2000). "Inovação: novos produtos, serviços e neócios para Portugal”. Ed. Verbo. Lisboa.

FRIED, D. (2011): "Afrontamiento generativo de crisis y conflictos en organizaciones". Revista Persona, no 14, págs.: 11 - 40.

GARCIA, E. (2001). "Calidad de servicio en hoteles de Sol y playa". Editorial Síntesis. Valencia.

GONZÁLEZ, Y. (2010): “El conflicto organizacional: una solución constructiva”. Pensando Psicología. Revista de la Facultad de Psicología. Universidad Cooperativa de Colombia. Vol. 6, no 11, págs. 11- 27. 
GÓMEZ, G. (2013): "El conflicto en las Organizaciones y Mediación". Edición. Electrónica. Universidad Internacional de Andalucía.

GRANT, R. (1996). "Toward a Knowledge-Based Theory of the Firm". Strategic Management Journal, vol. 17, Winter Special Issue, págs. 109-122.

LACA, F.y ALZATE, R. (2004). "Estrategias de conflicto y patrones de decisión bajo presión de tiempo". Revista Internacional de Ciencias Sociales y Humanidades, SOCIOTAM, vol. XIV, núm. 1, págs.: 11 - 32. Universidad Autónoma de Tamaulipas. Ciudad Victoria.

MENDES, R. y VAREIRO, L. (2012). "Desigualdades de género nos salarios e na produtividade na indústia do turismo em Portugal". Revista Portuguesa do Estudos Regionais, no 29. Angra de Heroísmo.

MERINO, C. (2008). “Gestión estratégica de conflictos en ámbito empresarial: Transferencia desde la práctica de la mediación". I Forum Internacional de Mediaçao Empresarial.

MOORE, C. (1986). “El Proceso de Mediación”. Editorial GRANICA. 1995.

PARÍS, S. (2003). "La conflictología. Un aprendizaje positivo de conflictos". Reseña de "Aprender del conflicto. Conflictología y Educación" de Eduard Vinyamata. Convergencia. Revista de Ciencias Sociales, vol. 10, núm. 33, pp. 315- 328. Universidad Autónoma del Estado de México.

RAHIM, M.A. (1985): "A Strategy for Managing Conflict in Complex Organizations" Human Relations. Vol. 38, no 1, págs. 81 - 89. Publisher: Tavistock Institute of Human Relations; University of Michigan. Research Center for Group Dynamics, SAGE Publications.

RAHIM, M.a (2002): "Toward a theory of managin organizational conflicto". The International Journal of Conflict Management. Vol. 13, № 3, págs.: $206-235$.

SAN CRISTOBAL, S. (2013): "Sistemas alternativos de resolución de conflictos: negociación, conciliación, mediación, arbitraje, en el ámbito civil y mercantil”. Anuario Jurídico y Económico Escurialense, XLVI (2013) 39-62. 